\title{
THE POSITION REGULARITIES OF FLARES RELATED TO THE FIELD MAXIMUM IN SUNSPOT GROUPS
}

\author{
V. V. KASINSKY \\ Siberian Institute of Terrestrial Magnetism, Ionosphere and Radio Propagation, Irkutsk, U.S.S.R.
}

\begin{abstract}
In this paper common regularities in flare positions have been studied in relation to spots as the extreme points of the magnetic field. The angular distribution diagrams of flares reveal that equatorial-western shifts of flares occurred during the I.G.Y. period (1957-58) in both hemispheres. This shift with some peculiarities is confirmed in the material of the 19th solar cycle, using the Solar Data bulletins, U.S.S.R., 1955-66. One result is that the western component of the shift does not change its sign and increases steadily during the cycle, while the equatorial component reverses sometimes and decreases steadily in amplitude to the end of the cycle.
\end{abstract}

A flare is associated with neutral points (or lines) of magnetic field. On the other hand an active region is a region of enhanced magnetic field. Sunspots are regions where a magnetic field has its maximum strength. The position of a spot unlike a neutral line is always fixed strictly, therefore in this paper common regularities in flare positions referring to spots as the extreme points of the field - center of the spots - have been studied.

Mean distance spot - flare is quite short (Waldmeier, 1938) therefore a principal piece of information about flare positions is the angular distribution of them related to the magnetic poles. The material used for this study was the synoptic maps of solar activity containing sketches of flares of importance $1+$ and higher at moments close to the maximum brightness in $\mathrm{H} \alpha$ (I.G.Y. Maps, 1961).

The angular distribution diagrams are drawn by counting the occurrence of flares in a discrete number of sectors arranged around the spot and then a summation over all spots. Flares of the northern and southern hemispheres are analyzed separately. Analysis of the diagrams leads to the conclusion that flares in the northern hemisphere seem to show a concentration southward while the southern ones are concentrated northward in relation to their centers (spots). It may be said that there is an equatorial shift of flares compared to spots in both hemispheres. It seems that this asymmetry is conserved for the strongest flares of importance $2+$ and 3 as well as for the leader and the follower spots in bipolar groups. (See Figure 1 and Figure 2.)

Analysis of the diagrams reveals that a western shift of flares is also present as it is noticed for some diagrams. To summarize over all spots, one can say that there is an equatorial - western shifting tendency of the position of flares during 1957-58.

The measure of positional asymmetry can be estimated by the formulae:

$$
P=\frac{n_{\mathrm{N}}-n_{\mathrm{S}}}{\sqrt{2\left(n_{\mathrm{N}}+n_{\mathrm{S}}\right)}} \quad Q=\frac{n_{\mathrm{E}}-n_{\mathrm{W}}}{\sqrt{2\left(n_{\mathrm{E}}+n_{\mathrm{W}}\right)}},
$$

where $n_{i}(i=\mathrm{N}, \mathrm{S}, \mathrm{E}, \mathrm{W})$ in the number of flares moving in each direction, the quan-

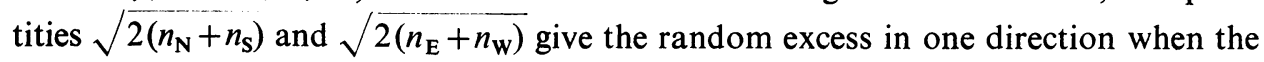


normal law distribution for excesses is assumed. If $P>1, Q>1$ the excess may be qualified as nonrandom and this is fulfilled for most cases.

These results are verified with the material of solar cycle 19 with about 12000 flares using the Solar Data bulletin (1954-1966, Nauka, U.S.S.R.). The relative positions are found by means of coordinates for flares and sunspots with an accuracy of $\pm 2^{\circ}$.

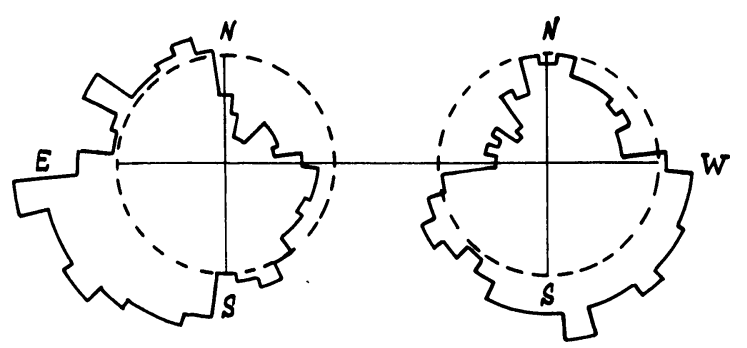

Fig. 1. The angular distribution of flares relative to the leader and follower spots in the northern hemisphere. Broken circle represents equable distribution.

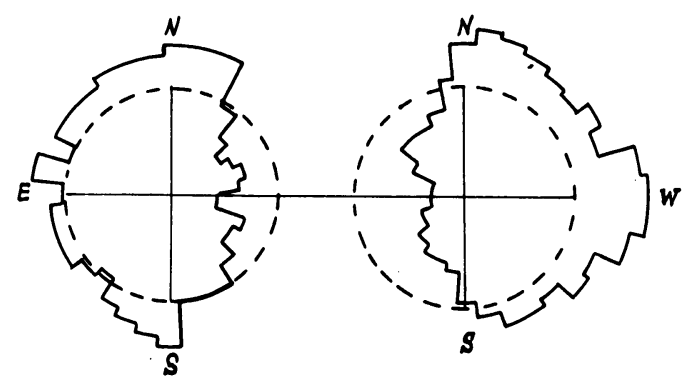

Fig. 2. The angular distribution of flares relative to the leader and follower spots in southern hemisphere.

The values of the asymmetries $P(t)$ and $Q(t)$ averaged over each month are plotted as a function of the time. $P(t)$ and $Q(t)$ show in such a run that on the average during the cycle the equatorial-western shifting tendency of flare positions seems to be confirmed, although sometimes an equatorial asymmetry changes its sign to the opposite (to the pole). This change occurred in the beginning of the 19th cycle in the southern hemisphere and in the beginning of the 20th cycle in the northern one.

It is significant to note that the equatorial shift reverses sometimes. Plots of functions $P$ and $Q$ also revealed the 'impulsive' character of the asymmetries especially noticeable when the numerical differentiation of $P(t), Q(t)$ with respect to time is taken. A halfwidth of impulses is of the order of $0.5-1$ year. On the other hand, by numerical integration of the asymmetry values one can show that the cumulative quantities $\sum P(t)$ and $\sum Q(t)$ grow significantly toward the end of the cycle. Cumulative asymmetries 
are reproduced in Table I where in the second column each hemisphere probability, assuming a normal law of distribution for excesses of flares, is given. It can be seen from the table that probabilities are negligible, that is, the asymmetry of flare positions is nonrandom in character.

\section{TABLE I}

\begin{tabular}{lrrrr}
$\Sigma P$ & -12.8 & $10^{-66}$ & +2.1 & $310^{-2}$ \\
$\Sigma Q$ & -8.9 & $10^{-34}$ & -8.0 & $10^{-27}$ \\
\hline Northern hemisphere & & \multicolumn{2}{l}{ Southern hemisphere }
\end{tabular}

Another interesting peculiarity of the flare positions follows from vector-diagrams if the vector shift of the center distribution of flares is plotted against time. For this mean year vector diagram it can be seen that the equatorial shift component increases to the maximum and decreases to the end of the cycle, while the western shift component increases steadily from the beginning to the end of the cycle.

The peculiarities in flare positions are difficult to interpret in terms of the wellknown magnetic field behavior. Perhaps it is connected with the geometry and development of large scale magnetic fields. But independent of the hypothetical reasons, the flare positions compared to field maximum points yield no less information than their positions relative to the zero points of the magnetic field.

\section{References}

I.G.Y. Solar Activity Maps: 1961, Di, v xx, Ann., I.G.Y. Pergamon Press.

Solar Data bulletin, Nauka, U.S.S.R. 1954-1966.

Waldmeier, M.: 1938, Z. Astrophys. 10, 276.

\section{Discussion}

Meyer: If the occurrence of flares is spatially correlated with the 'neutral-line' in active regions, one also expects an asymmetry of the kind you discussed, due to the systematic difference in latitudinal position and concentration between leading and following polarities in sunspot groups. Does the observed systematic distribution of flares that you find correspond to such an effect or is it at variance with it?

Kuklin: If the flares are concentrated near the zero-line between leading and following sunspots then the distribution asymmetry will be opposite for these two centers. However Figures 1 and 2 show the similarity of the distribution asymmetries as for the leading sunspot so for the following one in both hemispheres. Therefore the mentioned agreement is absent. 